\title{
Effects of Acute Hypoxic Provocation on Autonomic Nervous System in Young "Healthy" Smokers Measured by Heart Rate Variability
}

\author{
Zdravko Taralov ${ }^{1, *}$, Peter Dimov ${ }^{1}$, Kiril Terziyski ${ }^{1}$, Blagoi Marinov ${ }^{1}$ Mariyan \\ Topolov $^{2}$, Stefan Kostianev ${ }^{1}$
}

1. Department of Pahophysiology, Faculty of Medicine, Medical University Plovdiv, 15A Vasil Aprilov blvd, Plovdiv 4002, Bulgaria; pdimov@pathophysiology.info 2. Department of Pharmacology and Drug Toxicology, Faculty of Pharmacy, Medical University Plovdiv, 15A Vasil Aprilov blvd, Plovdiv 4002, Bulgaria; mtopolovmd@gmail.com

*Correspondence: ztaralov@pathophysiology.info; Tel: +359895193686

\section{Abstract}

Background and objectives: Smoking leads to autonomic dysfunction. However, the clinical methods for diagnosing this dysfunction are not sufficient. Since exogenous hypoxia leads to changes in the autonomic cardiac control, the aim of our study was to compare the activity of the autonomic nervous system via heart rate variability (HRV) in young "healthy" smokers and non-smokers before, during and after a short-term exogenous hypoxic exposure. Methods: Twenty-one healthy non-smoking males aged $28.0 \pm 7.4$ (mean $\pm S D$ ) and fourteen healthy smoking males aged 28.1 \pm 4.3 with 9.2 \pm 5.6 pack-years were subjected to one-hour hypoxic exposure $\left(\mathrm{F}_{\mathrm{i}} \mathrm{O}_{2}=12.3 \pm 1.5 \%\right)$ via hypoxicator (AltiPro 8850 Summit+, Altitude Tech, Canada) with simultaneous recording of electrocardiography and pulse oximetry. HRV data was derived via specific software (Kubios HRV, Finland) by analyzing the pre-hypoxic, hypoxic and post-hypoxic periods. Results: Standard deviation of the intervals between normal beats (SDNN) was higher in non-smokers in the pre-hypoxic period (62.0 \pm 32.1 vs $40.3 \pm 16.2, p=0.013)$ but not in hypoxia (75.7 \pm 34.8 vs $57.9 \pm 18.3, p=0.167)$. When comparing intragroup HRV changes of shifting from hypoxic to post-hypoxic (normoxic) conditions we found that there is a significant increase in the root mean square of successive RR interval differences (RMSSD) (65.9 \pm 40.2 vs $75.1 \pm 45.9, \mathrm{p}=0.011)$ and in the high frequency $(\mathrm{InHF})(6.8 \pm 1.4$ vs $7.2 \pm 1.3, p=0.014)$ and a decrease in LF/HF (3.0 \pm 2.3 vs $1.9 \pm 1.5, p<0.001)$, but these changes were observed only in the group of non-smokers. Conclusions: Smoking likely impairs autonomic regulation in young healthy males and may lead to a decreased HRV even before subjective clinical signs and symptoms. Hypoxic exposure test could be 
applied in clinical practice for early detection of autonomic dysfunction in smokers, because their parasympathetic reactivation is blunted when shifting from hypoxic to normoxic ambient conditions measured by HRV.

Keywords: heart rate variability, smoking, hypoxia, autonomic dysfunction

\section{Introduction}

Tobacco smoking is one of the most widespread bad habits in the world, especially in the countries of Eastern Europe. More than half of the male Bulgarian population aged 20-44 are smokers [1]. It is well known that tobacco smoke impairs respiratory and cardiovascular systems and is associated with diseases with highest burden, such as coronary artery disease (CAD), stroke, sudden cardiac death, chronic obstructive pulmonary disease, (COPD) etc. Some of the pathological conditions mentioned above are considered a result of the impaired autonomic regulation affected by smoking [2]. It has been proven that nicotine could increase the activity of the sympathetic nervous system (SNS) and plasma catecholamines' levels. Thus, it can cause tachycardia and vasoconstriction which can contribute to cardiovascular morbidity [3].

Heart rate variability (HRV) is a commonly used method for assessment of autonomic dysfunction [4-6]. Manzano et al. established that smoking leads to an acute alteration in the autonomic control, associated with sympathetic overactivation and diminished vagal activity. These alterations in the autonomic nervous system (ANS) continue for approximately 30 minutes after a single smoked cigarette [7]. Regular smoking also leads to long-term effects such as permanent sympathetic predominance (decreased overall variability and increased LF/HF index) in subjects without significant health complaints and 15 pack-years of smoking on average [8, 9]. Smokers also have decreased reactivity to vagal stimulation tests such as the Valsalva manoeuvre. This could be associated with the decreased baroreflex sensitivity, which normally leads to parasympathetic activation $[10,11]$. 
There is frequent speculation in alpine practice that smokers are more resistant to hypoxia as a result of the daily hypoxic "training" while smoking. This statement is disputable, since nicotine does not increase the hypoxic ventilatory response (HVR) and peripheral chemoreceptor sensitivity (PCS) [12].

Still, there is no widely accepted consensus about the effects of smoking on the autonomic control at hypoxic exposure and more research is needed. Furthermore, there is no data about the activity of the ANS after removal of the hypoxic factor.

The aim of our study was to compare the activity of the ANS via HRV in young "healthy" smokers and non-smokers before, during and after a short-term exogenous hypoxic exposure.

\section{Material and methods}

Twenty one healthy male non-smokers and 16 male "healthy" smokers with 9.2 \pm 5.6 (Mean \pm SD) pack-years were included in the study. All the subjects were having regular physical activity and no clinical complaints or diagnosis. Groups' age and antropometric data are shown in Table 1. Two of the tested subjects from the smoking group were excluded from the analysis due to more than $15 \%$ premature cardiac contractions from the whole record. Thus, 14 smokers completed the study protocol.

The subjects received all relevant information about the study including its aim, protocol, tests included and any potential side effects. A signed informed consent was obtained from all the subjects prior to inclusion in the study and a questionnaire about their physical status completed. During the experimental session, the participants did not take any medications, coffee, alcohol nor tobacco at least 2 hours prior to the test. A physical examination was carried out, including an electrocardiogram (ECG) which was reviewed by a cardiologist to exclude cardiovascular abnormalities or any rhythm or conductive disorders. No side effects or complaints were reported during the visit. The subjects were situated in a supine position, in a comfortable bed, in a quiet, well-aerated 
room with constant light and ambient temperature in the absence of any distracting factors. They were instructed to stay calm, without excessive voluntary movements or speaking and to breathe normally. During the first $10 \mathrm{~min}$ of the visit the participants breathed ambient air at altitude of $130 \mathrm{~m}$ (Plovdiv, Bulgaria). Subsequently, air with an oxygen concentration of $12.3 \pm 1.5 \%$ corresponding to altitude of about $4200 \mathrm{~m}$ was administered for one hour via full-face mask using a hypoxicator (AltiPro 8850 Summit+, Altitude Tech, Canada). The protocol did not include any changes in the barometric pressure (normobaric hypoxia). After the end of the hypoxic session the mask was removed and the subjects were exposed to ambient air. Four-channel ECG ( $\mathrm{H}^{+}+$, Mortara Instruments, Milwaukee, USA) and pulse oximetry (CMS50F, Contec Medical Systems, Qinhuangdao, China) were recorded during the whole visit. ECG recordings were reviewed and R-R intervals were extracted automatically by $\mathrm{H}$-Scribe 5 software (Mortara Instruments, Milwaukee, USA).

Five-minute samples were selected from the end of the pre-hypoxic period, the end of hypoxia and immediately after the hypoxic exposure for the subsequent analysis. After removing trends, data was analyzed using Kubios HRV software [13] by which time domain, frequency domain and non-linear parameters were calculated. Fast Fourier transform was used for calculation of the frequency domain parameters using Welch's periodogram with a window length of $256 \mathrm{~s}$ and $50 \%$ overlap. The following parameters were derived from the RR data: Total Power (TP) and standard deviation of the intervals between normal beats (SDNN) as measures of overall autonomic regulation; absolute power and normalized units $(\mathrm{nu})$ of high frequency (HF; $0.15-0.40 \mathrm{~Hz}$ ) and low frequency (LF; $0.04-0.15 \mathrm{~Hz})$ spectral components, respectively reflecting parasympathetic nervous system (PNS) activity and combined sympathetic (SNS) and PNS activities. The ratio LF/HF was also calculated as an index of sympatho-vagal balance. Root mean square of successive R-R interval difference (RMSSD) is a time domain parameter associated with the parasympathetic activity. In addition to the linear 
methods described above, three commonly used nonlinear parameters were applied. These include standard deviations SD1 and SD2 of the Poincaré plot - SD1 related to fast beat-to-beat variability in data and SD2 describing longer-term variability of $R-R$ $[14,15]$. Sample Entropy (SampEn) was also collated as an index for the overall complexity and predictability of HRV time series [16].

Statistical analysis was performed by Independent Sample T-test (SPSS v.17.0) when comparing the group of smokers and non-smokers and Paired Sample T-test was used to compare intragroup changes between hypoxic and post-hypoxic periods. Normality of distribution was assessed by Shapiro - Wilk test. Skewness of distribution of some parameters (absolute spectral powers) was normalized by means of natural logarithmic transformation.

Official permission for the study was obtained by the Medical University of Plovdiv Ethics Committee with a protocol ref. number P-1548/13.03.2014. Guidelines for proper Human care were fully implemented following the Helskinki agreement and human rights.

\section{Results}

There was no difference in $\mathrm{SpO}_{2}$ between smokers and non-smokers in normoxic conditions. However, smokers had significantly higher oxygen saturation during the hypoxic exposure, but lower in the post-hypoxic period. They also had higher mean heart rate during the whole visit compared to non-smokers. HRV analysis showed that SDNN was higher in non-smokers in the pre-hypoxic period but not in hypoxia. RMSSD, SD1, SD2 и SD1/SD2 were higher in non-smokers during all three periods of the visit. Sample Entropy was higher in the group of non-smokers in normoxic but not in hypoxic conditions (Table 2).

Frequency domain HRV analysis showed higher TP in non-smokers only during the prehypoxic period. HF was lower in the smokers' group in both normoxic and hypoxic conditions. LF was also lower but only during the pre-hypoxic normoxia. Non-smokers 
had lower LF/HF index in normoxic (pre- and post-hypoxia) conditions but no significant difference was found during hypoxic exposure (Fig. 1).

When comparing intragroup changes of HRV of shifting from hypoxic to post-hypoxic (normoxic) conditions we found that there is a significant increase in SDNN, RMSSD and $\mathrm{HF}$ and a decrease in LF/HF, but these changes were observed only in the group of non-smokers (Table 3).

Peak frequency for HF at the three different stages of the study was as follows: before $(0.21 \pm 0.06)$, during $(0.21 \pm 0.07)$ and after hypoxia $(0.21 \pm 0.05)$ and did not show significant difference $(p=1.000)$.

\section{Discussion}

When comparing groups of smokers and non-smokers in normoxic conditions before hypoxic exposure there is no difference in the oxygen saturation, but smokers have significantly higher heart rate and lower HRV. It means that tobacco smoking leads to a decrease in the overall variability (Total power and SDNN), lower vagal activity (RMSSD, HF and SD1), sympathetic predomination (higher LF/HF and lower SD1/SD2) (Fig. 1) and lower complexity of the time series (Sample Entropy).

Impaired autonomic regulation in smokers has been studied before. Manzano et al. found that smoking of a single cigarette is a stress for the body and leads to a sudden change in the autonomic control associated with sympathetic activation and vagal withdrawal. These changes could be observed up to 30 minutes after smoking [7]. In our study we tried to avoid the effect of acute tobacco smoking, therefore our subjects did not smoke at least two hours prior to the test. Thus, we were able to assess the long-term consequences caused by smoking.

The results of our study are in concordance with other investigations that found impaired autonomic control with lower HRV parameters in "heavy" smokers with more 
than 15 pack-years [8, 9]. Although, our subjects were much younger and with less pack-years, we found the same HRV pattern. Even without any clinical signs and symptoms smokers had sympathetic predomination (higher LF/HF and lower SD1/SD2). Thus, HRV could be used in clinical practice for assessment of the impaired autonomic control in young smokers. Moreover, some studies show that HRV parameters significantly increase and LF/HF decrease after smoking cessation which clearly demonstrates the benefit of cessation [17].

When comparing the groups during exogenous hypoxic exposure we found that smokers had higher $\mathrm{SpO}_{2}$ and $\mathrm{HR}$ but significantly lower HRV parameters (RMSSD, HF, SD1, SD2, SD1/SD2) which means higher sympathetic activation as a result of hypoxic provocation. Higher $\mathrm{SpO}_{2}$ in smokers could not be a result of higher ventilation compared to non-smokers since nicotine does not increase the peripheral chemoreceptor sensitivity [12] and there was no difference in the peak frequency of HF, which suggests no difference in the respiratory rate of the two groups [18].

Moreover, it is well known that chronic hypoxia causes pulmonary vasoconstriction and pulmonary hypertension, which leads to ventilation/perfusion mismatch and remodeling of the pulmonary vessels $[19,20]$. Brewer et al. demonstrated that smoking workers of Leadville, Colorado $(3048 \mathrm{~m})$ had higher hemoglobin affinity to oxygen compared to nonsmokers [21], most probably caused by the higher levels of carboxyhemoglobin, that shifts the oxygen dissociation curve to the left [22]. This fact could be a possible explanation of the higher $\mathrm{SpO}_{2}$ of smokers in hypoxia but not in normoxia, since the difference in the oxygen dissociation curve of the two groups is higher in lower $\mathrm{pO}_{2}$ (high altitude) [23]. However, carboxyhemoglobin levels were not part of the study design and further tests are needed to confirm this hypothesis.

The shift from hypoxic to normoxic conditions in non-smokers was associated with a sudden increase of $\mathrm{SpO}_{2}$ and overall variability (SDNN) caused by parasympathetic 
activation (RMSSD, InHF) (Fig. 2), which is normally observed in the recovery period in subjects with good autonomic control like athletes [24]. Conversely, there was no change of HRV parameters in smokers and the LF/HF index even increased which was evident for demonstrating the absence of parasympathetic response when the hypoxic stimulus is removed. The absence of autonomic response is most probably a result of the autonomic dysfunction in smokers, previously described in the literature [9, 25].

There is no sufficient data about the changes in the activity of the autonomic nervous system when shifting from hypoxic to normoxic environment. Since transition from hypoxic to normoxic conditions can induce PSNS activation which is not observed in smokers, our hypoxic provocation protocol could be applied in the clinical practice as a test for early detection of autonomic dysfunction in smokers, even before the appearance of clinical signs.

However, some study limitations of our research should be considered. We did not record the respiratory rate and tidal volume, therefore we do not have information about ventilation that is known to affect the HRV parameters [15]. However, the peak frequency of HF (which is suggested as an indirect index of respiratory rate [18]) showed no changes during the different periods of the protocol. Thus, we assume that ventilation of examined subjects was stable.

The sample of the study is not big enough to produce more explicit results. Increasing the number of enrolled subjects would potentially decrease the impact of confounding factors affecting HRV.

\section{Conclusion}

In conclusion, the results of our study showed that smoking impairs the autonomic modulation in young "healthy" smokers and leads to a decreased HRV even before the appearance of any subjective clinical signs and symptoms. During acute exposure to exogenous hypoxia smokers have higher $\mathrm{SpO}_{2}$ but lower HRV parameters - RMSSD, 
InLF, InHF and Poincaré plot standard deviations SD1, SD2 and SD1/SD2 index. Data suggests that smokers have altered autonomic regulation under hypoxic conditions diminished parasympathetic activity and sympathetic domination despite having higher $\mathrm{SpO}_{2}$. Transition from hypoxic to normoxic conditions leads to an-increase in the PSNS activity, which was observed only in the group of non-smokers. Therefore, hypoxic exposure test could be applied in clinical practice for early detection of autonomic dysfunction in smokers, because their parasympathetic reactivation is blunted when shifting from hypoxic to normoxic ambient conditions measured by HRV.

Table 1. Comparison between age and main antropometric parameters of the tested groups

\begin{tabular}{|l|l|l|l|}
\hline Groups & Non- & Smokers & p \\
\hline Age (years) & $28.0 \pm 7.4$ & $28.1 \pm 4.3$ & 0.949 \\
\hline Height (cm) & $179.9 \pm 6.2$ & $179.7 \pm 4.3$ & 0.941 \\
\hline Weight (kg) & $78.3 \pm 6.6$ & $77.4 \pm 10.0$ & 0.762 \\
\hline Body Mass Index & $24.3 \pm 2.4$ & $24.0 \pm 3.2$ & 0.796 \\
\hline
\end{tabular}

Table 2. Comparison of oxygen saturation, heart rate and HRV parameters between "healthy" smokers and non-smokers before, during and after exogenous hypoxia provocation.

\begin{tabular}{|l|l|l|l|l|l|l|l|l|l|}
\hline Groups & Non- & Smokers & & Non- & Smokers & & Non- & Smokers \\
\hline smokers & & & smokers & & smokers & \\
\hline Period & hefore & Before & $\mathbf{p}$ & Hypoxia & Hypoxia & $\mathbf{p}$ & After & After & $\mathbf{p}$ \\
\hline $\mathrm{SpO}_{2}(\%)$ & $96.8 \pm 1.2$ & $96.3 \pm 1.6$ & 0.276 & $88.2 \pm 5.9$ & $91.4 \pm 1.4$ & $\mathbf{0 . 0 2 6}$ & $96.8 \pm 1.1$ & $95.9 \pm 1.1$ & $\mathbf{0 . 0 2 1}$ \\
\hline $\mathrm{HR}_{(\text {beat.min }}$ ) & $62.2 \pm 9.0$ & $79.3 \pm 14.2$ & $\mathbf{0 . 0 0 8}$ & $65.1 \pm 11.1$ & $77.3 \pm 13.8$ & $\mathbf{0 . 0 0 7}$ & $61.9 \pm 9.0$ & $74.5 \pm 13.5$ & $\mathbf{0 . 0 0 6}$ \\
\hline SDNN (ms) & $62.0 \pm 32.1$ & $40.3 \pm 16.2$ & $\mathbf{0 . 0 1 3}$ & $75.7 \pm 34.8$ & $57.9 \pm 18.3$ & 0.167 & $87.8 \pm 39.2$ & $61.9 \pm 9.0$ & $\mathbf{0 . 0 2 1}$ \\
\hline
\end{tabular}




\begin{tabular}{|l|l|l|l|l|l|l|l|l|l|}
\hline RMSSD $(\mathrm{ms})$ & $59.3 \pm 32.8$ & $24.9 \pm 12.5$ & $<\mathbf{0 . 0 0 1}$ & $65.9 \pm 40.1$ & $34.3 \pm 17.1$ & $\mathbf{0 . 0 0 3}$ & $75.1 \pm 45.9$ & $34.9 \pm 20.0$ & $\mathbf{0 . 0 0 1}$ \\
\hline InTP $\left(\mathrm{ms}^{2}\right)$ & $7.7 \pm 1.1$ & $7.0 \pm 0.8$ & $\mathbf{0 . 0 4 6}$ & $8.2 \pm 1.7$ & $7.9 \pm 1.0$ & 0.309 & $8.5 \pm 0.9$ & $7.9 \pm 0.9$ & 0.068 \\
\hline InLF $\left(\mathrm{ms}^{2}\right)$ & $6.9 \pm 1.1$ & $6.0 \pm 1.0$ & $\mathbf{0 . 0 1 5}$ & $7.6 \pm 1.1$ & $7.1 \pm 0.9$ & 0.152 & $7.6 \pm 0.9$ & $7.0 \pm 0.8$ & 0.089 \\
\hline InHF $\left(\mathrm{ms}^{2}\right)$ & $6.5 \pm 1.5$ & $5.3 \pm 1.1$ & $\mathbf{0 . 0 1 3}$ & $6.8 \pm 1.4$ & $5.8 \pm 1.6$ & $\mathbf{0 . 0 3 4}$ & $7.2 \pm 1.3$ & $5.8 \pm 1.1$ & $\mathbf{0 . 0 0 2}$ \\
\hline LF/HF & $1.4 \pm 0.8$ & $2.3 \pm 0.9$ & $\mathbf{0 . 0 4 0}$ & $3.0 \pm 2.3$ & $3.8 \pm 1.6$ & 0.273 & $1.9 \pm 1.5$ & $4.0 \pm 1.8$ & $\mathbf{0 . 0 0 1}$ \\
\hline SD1 (ms) & $41.9 \pm 23.2$ & $19.4 \pm 12.4$ & $\mathbf{0 . 0 0 1}$ & $42.9 \pm 23.4$ & $22.2 \pm 9.0$ & $\mathbf{0 . 0 0 1}$ & $53.6 \pm 32.7$ & $24.7 \pm 14.2$ & $\mathbf{0 . 0 0 1}$ \\
\hline SD2 (ms) & $93.7 \pm 39.9$ & $53.3 \pm 19.2$ & $<\mathbf{0 . 0 0 1}$ & $120.6 \pm 39.0$ & $85.0 \pm 23.9$ & $\mathbf{0 . 0 0 2}$ & $144.5 \pm 49.1$ & $87.7 \pm 32.6$ & $\mathbf{0 . 0 0 1}$ \\
\hline SD1/SD2 & $0.43 \pm 0.10$ & $0.34 \pm 0.10$ & $\mathbf{0 . 0 1 8}$ & $0.33 \pm 0.10$ & $0.26 \pm 0.06$ & $\mathbf{0 . 0 0 5}$ & $0.35 \pm 0.11$ & $0.26 \pm 0.10$ & 0.071 \\
\hline SampEn & $1.6 \pm 0.2$ & $1.4 \pm 0.3$ & $\mathbf{0 . 0 4 9}$ & $1.4 \pm 0.2$ & $1.3 \pm 0.2$ & 0.233 & $1.3 \pm 0.2$ & $1.2 \pm 0.2$ & $\mathbf{0 . 0 2 1}$ \\
\hline
\end{tabular}

Legend: HF - high frequency, HR - heart rate, LF - low frequency, SD - standard deviation, SDNN - standard deviation of the intervals between normal beats, $\mathrm{SpO}_{2}-$ oxygen saturation, RMSSD - root mean square of successive RR interval differences, SampEn - sample entropy, TP - total power

Table 3. Comparison of HRV parameters between hypoxic and normoxic (post-hypoxic) conditions in smokers and non-smokers

\begin{tabular}{|c|c|c|c|c|c|c|}
\hline \multirow{2}{*}{$\begin{array}{l}\text { Groups } \\
\text { Parameters }\end{array}$} & \multicolumn{2}{|c|}{ Non-smokers } & \multirow[b]{2}{*}{ p } & \multicolumn{2}{|c|}{ Smokers } & \multirow[b]{2}{*}{$\mathbf{p}$} \\
\hline & Hypoxia & $\begin{array}{l}\text { Post- } \\
\text { Hypoxia }\end{array}$ & & Hypoxia & $\begin{array}{l}\text { Post- } \\
\text { Hypoxia }\end{array}$ & \\
\hline HR (beat.min-1) & $65.1 \pm 11.1$ & $61.9 \pm 9.0$ & 0.001 & $77.3 \pm 13.8$ & $74.5 \pm 13.5$ & 0.033 \\
\hline SDNN (ms) & $75.7 \pm 41.1$ & $87.8 \pm 39.2$ & 0.055 & $57.9 \pm 28.1$ & $61.3 \pm 25.3$ & 0.656 \\
\hline RMSSD (ms) & $65.9 \pm 40.2$ & $75.1 \pm 45.9$ & 0.011 & $34.3 \pm 17.1$ & $34.9 \pm 20.0$ & 0.914 \\
\hline $\operatorname{lnLF}\left(\mathrm{ms}^{2}\right)$ & $7.6 \pm 1.1$ & $7.6 \pm 0.9$ & 0.963 & $7.1 \pm 0.9$ & $7.0 \pm 0.8$ & 0.983 \\
\hline $\operatorname{lnHF}\left(\mathrm{ms}^{2}\right)$ & $6.8 \pm 1.4$ & $7.2 \pm 1.3$ & 0.014 & $5.8 \pm 1.2$ & $5.8 \pm 1.1$ & 0.912 \\
\hline $\operatorname{lnTP}\left(\mathrm{ms}^{2}\right)$ & $8.2 \pm 1.2$ & $8.5 \pm 0.9$ & 0.099 & $7.9 \pm 1.0$ & $7.9 \pm 0.9$ & 0.785 \\
\hline $\mathrm{LF} / \mathrm{HF}$ & $3.0 \pm 2.3$ & $1.9 \pm 1.5$ & $<0.001$ & $3.8 \pm 1.6$ & $4.0 \pm 1.8$ & 0.657 \\
\hline
\end{tabular}

Legend: HF - high frequency, HR - heart rate, LF - low frequency, SDNN - standard deviation of the intervals between normal beats, RMSSD - root mean square of successive RR interval differences, TP - total power 


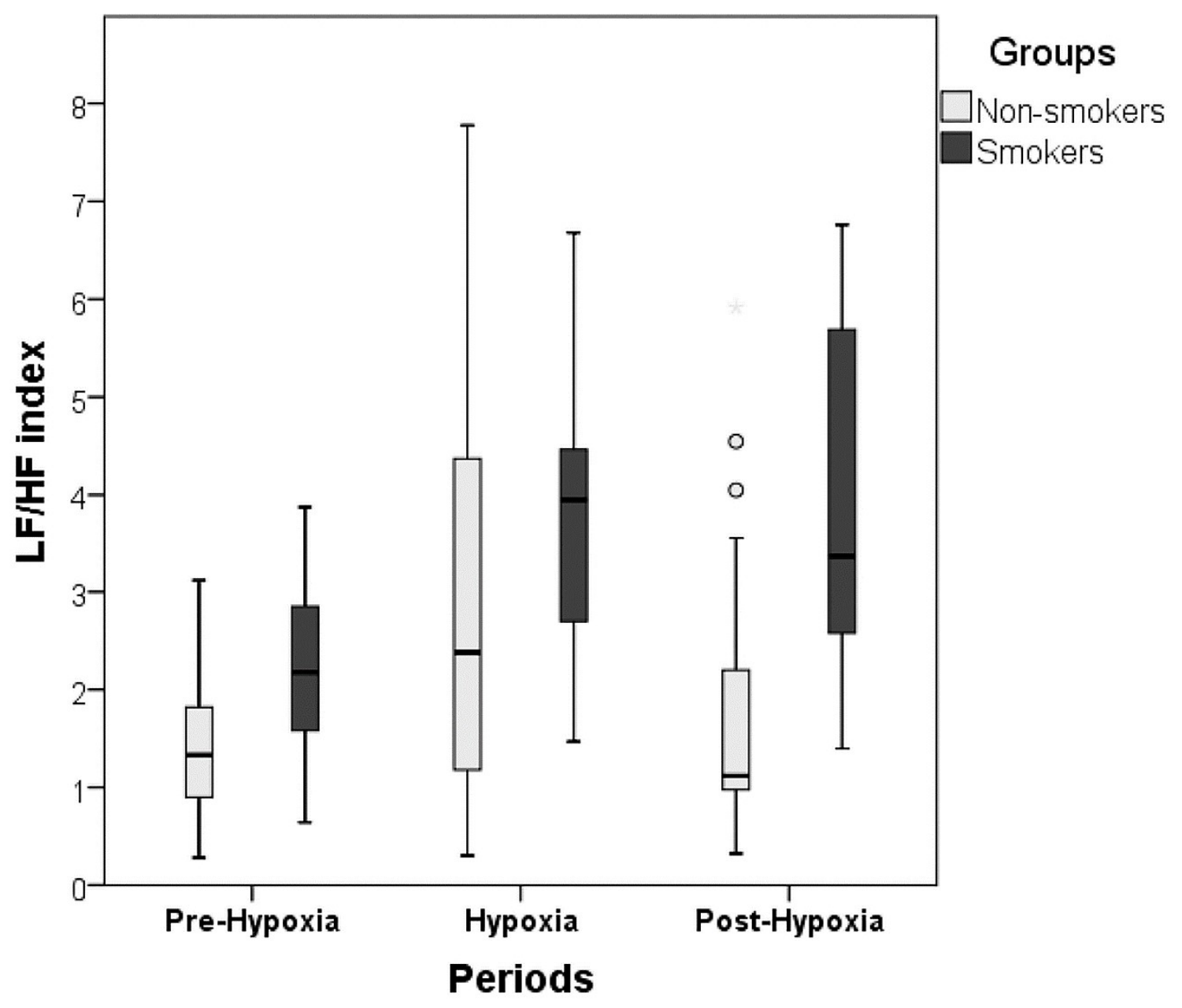

Figure 1. Comparison of LF/HF index between smokers and non-smokers in normoxic and hypoxic conditions. 


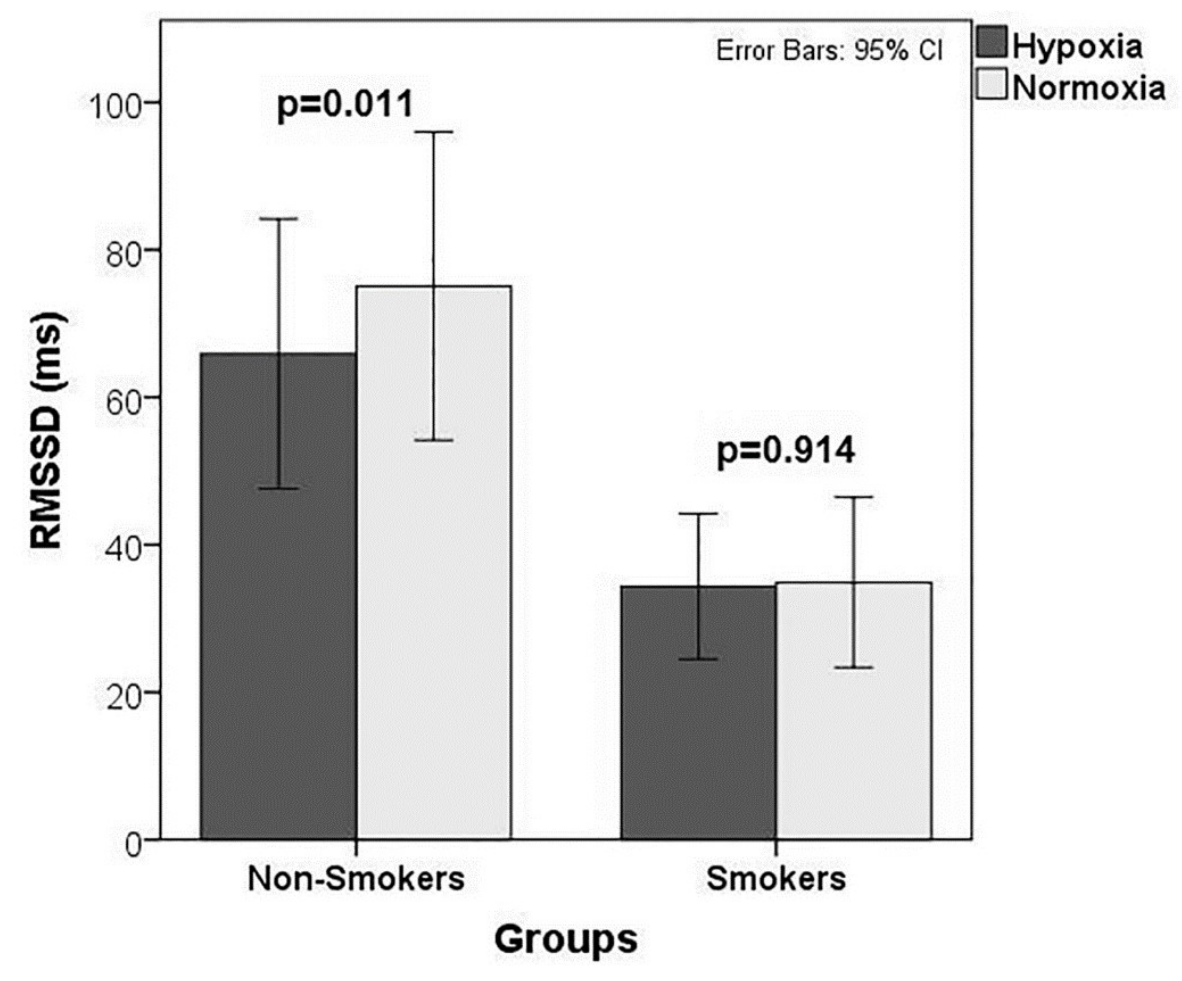

Figure 2. Comparison of RMSSD between non-smokers and smokers in hypoxic and posthypoxic (normoxic) ambient conditions

\section{Authors contribution}

Conceptualization: Z.T. and K. T..; Methodology: Z. T. and B. M..; Software: K. T. and ZT.; Validation: B.M. and S.K.; Formal Analysis: P.D. and Z.T..; Investigation: Z.T. and P.D..; Resources: M.T.,; Data Curation: Z.T., P.D. and M.T.; Writing: Z.T., P.D. and M.T.; Writing - Review \& Editing: K.T. and B.M.; Visualization M.T. and Z.T.; Supervision: S.K.

\section{Conflicts of interest}

The authors declare no conflict of interest.

\section{References:}


1. Zatonski, W.; Przewozniak, K.; Sulkowska, U.; West, R.; Wojtyla, A. Tobbaco smoking in countries of the European Union. Ann. Agric. Environ. Med. 2012, 19, 181-192.

2. Modala, S.; Ahmed, Q.R.; Sau, S.K. Effect on autonomic nervous system in smokers and non-smokers - a comparison study. Natl. J. Med. Allied Sci. 2012, $1,25-28$.

3. Papathanasiou, G.; Mamali, A.; Papafloratos, S.; Zerva, E. Effects of smoking on cardiovascular function: the role of nicotine and carbon monoxide. Health science journal. 2014, 8, 274-290.

4. Ferreira, M.; Zanesco, A. Heart rate variability as important approach for assessment autonomic modulation. Motriz: J. Phys. Ed. 2016, 22, 3-8.

5. Kang, J.H.; Kim, J.K.; Hong, S.H.; Lee, C.H.; Choi, B.Y. Heart rate variability for quantification of autonomic dysfunction in fibromyalgia. Ann. Rehabil. Med. 2016, 40, 301-309.

6. Sgoifo, A.; Carnevali, L.; Alfonso, Mde, L.; Amore, M. Autonomic dysfunction and heart rate variability in depression. Stress. 2015, 18, 343-352.

7. Manzano, B.M.; Vanderlei, L.C.; Ramos, E.M.; Ramos D. Acute effects of smoking on autonomic modulation: analysis by Poincare plot. Arq. Bras. Cardiol. 2011, 96, 154-60.

8. Behera, J,K,; Sood, S.; Gupta, R.; Kumar, N.; Singh, M.; Gupta, A. Assessing autonomic function in smokers. Australasian Medical Journal. 2010, 3, 712-715.

9. Ferdouse, M.; Ferdousi, S. Autonomic dysfunction in current cigarette smokers assessed by time series analysis of heart rate variability. J. Bangladesh Soc. Physiol. 2013, 8, 84-8.

10. Middlekauff, H.R.; Park, J.; Moheimani, R.S.; Adverse effects of cigarette and noncigarette smoke exposure on the autonomic nervous system. J. Am. Coll. Cardiol. 2014, 64, 1740-1750. 
11.Papathanasiou, G.; Georgakopoulos, D.; Papageorgiou, E.; Zerva, E.; Michalis, L.; Kalfakakou, V.; Evangelou, A. Effects of smoking on heart rate at rest and during exercise, and on heart rate recovery in young adults. Hellenic J. Cardiol. 2013, 54, 168-177.

12. Najem, B.; Houssiere, A.; Pathak, A.; Jansen, C.; Lemogoum, D.; Xhaet, O.; Cuylits, N.; van de Borne, P. Acute cardiovascular and sympathetic effects on nicotine replasement therapy. Hypertension. 2006, 47, 1162-1167.

13.Tarvainen, M.P.; Niskanen, J.P.; Lipponen, J.A.; Ranta-Aho, P.O.; Karjalainen, P.A. Kubios HRV - Heart rate variability analysis software. Comput. Methods Programs Biomed. 2014, 113, 210-220.

14.Rajendra, A.U.; Joseph, P.; Kannathal, N.; Lim, C.M.; Suri, J. Heart rate variability: A review. Med. Biol. Eng. Comput. 2007, 44, 1031-1051

15. Task force of The European Society of Cardiology and The North American Society of Pacing and Electrophysiology. Heart rate variability. Standards of measurement, physiological interpretation and clinical use. Circulation. 1996, 93, 1043-1065.

16. Voss, A.; Schulz, S.; Schroeder, R.; Baumert, M.; Caminal, P. Methods derived from nonlinear dynamics for analyzing heart rate variability. Philos. Trans. Royal Soc. A. 2009, 367, 277-96.

17. Minami, J.; Ishimitsu, T.; Matsuoka, H. Effects of smoking cessation on blood pressure and heart rate variability in habitual smokers. Hypertension. 1999, 33, 586-590.

18. Thayer, H.F.; Sollers, J.J. ${ }^{\text {rd }}$; Ruiz-Padial, E.; Vila, J. Estimating respiratory frequency from autoregressive spectral analysis of heart period. IEEE Eng. Med. Biol. Mag. 2002, 21, 41-45.

19. Ferrer, E.; Peinado, V.I.; Castaneda, J.; Prieto-Lloret, J.; Olea, E.; GonzalezMartin, M.C.; Vega-Agapito, M.V.; Domingues-Fandos, D.; Obeso, A.; Gonzalez, 
C.; Barbera, J.A. Effects of cigarette smoke and hypoxia on pulmonary circulation in the guinea pig. Eur. Respir. J. 2011, 38, 617-627.

20. Moudgil, R.; Michelakis, E.D.; Archer, S.L.; Hypoxic pulmonary vasoconstriction. J. Appl. Physiol. 2005, 98, 390-403.

21.Brewer, G.J.; Sing, C.F.; Eaton, J.W.; Weil, J.V.; Brewer, L.F.; Grover, R.F. Effects on hemoglobin oxygen affinity of smoking in residents of intermediate altitude. J. Lab. Clin. Med. 1974, 84, 191-205.

22. Freitas, J. Tobacco influence on carboxyhemoglobin, oxihemoglobin dissociation and erythrocyte filtration. Acta Med. Port. 1983, 4, 73-5.

23. West, J.B. The physiologic basis of high-altitude diseases. Ann. Intern. Med. 2004, 141, 789-800.

24. Imai, K.; Sato, H.; Hori, M.; Kusuoka, H.; Ozaki, H.; Yokoyama, H.; Takeda, H.; Inoue, M.; Kamada, T. Vagally mediated heart rate recovery after exercise is accelerated in athletes but blunted in patients with chronic heart failure. J. Am. Coll. Cardiol.. 1994, 24, 1529-1535.

25. Burtscher, M.; Szubski. C.; Faulhaber, M.; Prediction of susceptibility to AMS in simulated altitude. Sleep Breath 2008, 12, 103-108. 